Revista Latinoamericana de la Papa 21 (1): 1 - 14

http://www.papaslatinas.org/revista.html

ISSN: $1853-4961$

\title{
Escenarios bioclimáticos de tizón tardío [Phytophthora infestans (Mont.) De Bary] en Ciego de Ávila, Cuba
}

\author{
A. A. Hernández-Mansilla ${ }^{1 / *}$, R. Sorí-Gómez ${ }^{2}$, Y. Valentín-Pérez ${ }^{1}$, A. López- \\ Mayea $^{1}$, O. Benedico-Rodríguez ${ }^{1}$
}

Recibido: 03/03/2016

Aceptado: 23/10/2017

Accesible en línea: Diciembre 2017

\section{Resumen}

Constituye un quehacer relevante para la investigación agrícola actual predecir e interpretar la influencia del cambio climático sobre los organismos nocivos de los cultivos y adaptar el manejo fitosanitario. Interpretar los escenarios bioclimáticos de Phytophthora infestans, agente causal del Tizón tardío de la papa en áreas del cultivo en Ciego de Ávila durante el período de noviembre - abril para los años 2025 y 2030 bajo escenarios (SRES) A2 y B2 constituyó el objetivo de este trabajo. En la confección de los escenarios bioclimáticos se emplearon datos diarios de acumulados de precipitación y temperatura media del Modelo Regional PRECIS-CARIBE. Además de los índices biológicos requeridos por el fitopatógeno para desarrollar epifitias considerados en el método de "Umbral de lluvias" recomendado por el Instructivo técnico del cultivo en Cuba. Los resultados coinciden en un ambiente con condiciones meteorológicas no favorables para el desarrollo y la evolución de Tizón tardío tanto en 2025 como en 2030. Específicamente, la poca ocurrencia y al bajo acumulado de las precipitaciones que no superan los niveles que señala la línea de umbral, así como la incidencia de temperaturas medias superiores a $24{ }^{\circ} \mathrm{C}$. Los escenarios bioclimáticos obtenidos de este Oomycetes brindan información para la futura planificación y el manejo fitosanitario del cultivo; selección de insumos y atenciones culturales que propicien la aplicación de una agricultura climáticamente inteligente que contribuya a la producción sostenible del tubérculo.

Palabras claves adicionales: Escenarios bioclimáticos, Phytophthora infestans, enfermedades de papa, "agricultura climáticamente inteligente", calentamiento global.

\footnotetext{
* Autor para correspondencia. Correo electrónico: ahmansilla@gmail.com, alexis.hernandez@cav.insmet.cu

1 Centro Meteorológico Provincial Ciego de Ávila. Marcial Gómez, No.401, esquina Estrada. Ciego de Ávila. Cuba.

2 Environmental Physics Laboratory (EPhysLab), Facultad de Ciencias de Ourense, Universidad de Vigo, Ourense, España. CP: 32004.
} 


\section{Bioclimatic scenarios of Late blight in potato [Phytophthora infestans (Mont.) De Bary] in Ciego de Avila, Cuba}

\section{Summary}

It is a relevant task for current agricultural research to predict and interpret the influence of climate change on the harmful organisms of crops and to adapt the phytosanitary management. Interpreting the bioclimatic scenarios of Phytophthora infestans, which is the causal agent of late blight of potato in the crops areas in Ciego de Ávila during the period November - April for the years 2025 and 2030 under scenarios (SRES) A2 and B2 was the aim of this work. In the making of the bioclimatic scenarios, were used daily data of accumulated precipitation and the average temperature from the Regional Model PRECISCARIBE. In addition, the biological indexes required by the phytopathogen to develop epiphytics which are considered in the method of "threshold of rains" recommended by the technical instruction of the crop in Cuba were taken into account. The results agree on the existence of not favorable meteorological conditions for the development and evolution of late blight in both 2025 and 2030. Specifically, the scarce occurrence and the low cumulative of precipitations that do not exceed the levels indicated by the threshold line, as well as the incidence of average temperatures above $24{ }^{\circ} \mathrm{C}$. The bioclimatic scenarios obtained for this Oomycetes provide information for future planning and phytosanitary management of the crop as well as for the selection of cultural inputs and services that favor the application of climate-smart agriculture that contributes to the sustainable production of the tuber.

Additional keywords: Bioclimatic scenarios, Phytophthora infestans, potato diseases, climate smart agriculture, global warming.

\section{Introducción}

La agricultura en el siglo XXI se enfrenta al desafío de satisfacer las demandas de alimentos y al mismo tiempo cumplir con los objetivos de sostenibilidad (Lamichhane et al. 2014). Sin embargo, las evidencias del calentamiento global durante las últimas décadas y las grandes posibilidades de mayor ocurrencia de eventos climáticos extremos (olas de calor, sequías, inundaciones, etc.) (IPCC 2014) son factores que directa e indirectamente afectan la pérdida de la capacidad productiva, niveles productivos y con ello la seguridad alimentaria. El cambio climático es un fenómeno determinante en la producción alimenticia actual y futura por sus efectos sobre las biodiversidad agroproductiva; son reconocidas las alteraciones y cambios que ocurren en los sistemas naturales y manejados que muestran ya impactos en los ciclos estacionales y estados de vida (fenológicos) de las especies vegetales (Rosenzweig et al. 2007), destacándose los cambios anticipados de gran envergadura en las zonas tropicales de países en desarrollo (Cline 2007).

Existe una gran necesidad de desarrollar apropiados marcos de trabajo, por ejemplo, para orientar la planificación y priorización de la investigación sobre el cambio relacionada con plagas de los cultivos (Juroszek \& Tiedemann 2013). Y es que los rendimientos dependen en gran medida de los organismos nocivos que inciden en los sistemas de explotación agrícolas, estos son responsables de ocasionar pérdidas entre un $20 \%$ y $40 \%$ de la cosecha potencial fundamentalmente 
en países en desarrollo (UNEP 2009). En una revisión de estudios sobre impactos del cambio climático en organismos nocivos, Altieri \& Nicholls (2008) concluyen que los insectos plagas aumentarán en abundancia en la medida que la temperatura aumente. Igualmente señalan la posibilidad de extensión en los ciclos y cambios fenológicos; con respuestas de adaptación más rápidas para las plagas migratorias que para las plantas y las ventajas de colonización de nuevos cultivos y hábitats. Otros autores puntualizan que los efectos del cambio climático sobre los fitopatógenos en las plantas pueden ser positivos, negativos o neutrales, en dependencia de cada región o períodos (Ghini et al. 2008).

Estas consideraciones conducen a reflexionar sobre la manifestación de un impacto negativo del cambio climático sobre el rendimiento de los cultivos, su producción y el aumento de los precios de los alimentos que afectarán directamente al bienestar humano (Nelson et al. 2009). Existe una gran necesidad de desarrollar apropiados marcos de trabajo, por ejemplo, para orientar la planificación y priorización de la investigación sobre el cambio relacionada con plagas de los cultivos (Juroszek \& Tiedemann 2013).

El cultivo de la papa es un reglón económico y alimenticio de relevancia, importantes en la dieta y medios de subsistencia de millones de personas en todo el mundo (Luck et al. 2011). En Cuba, figura como uno de los productos del agro de mayor relevancia por su gran aceptación y demanda de sus habitantes que lo sitúan como uno de los componentes básicos de la canasta de consumo familiar. De esta forma, ocupa un lugar preponderante en la seguridad alimentaria en el país; razón por la cual, el Estado a través del Gobierno y el
Ministerio de la Agricultura le dedican una amplia gama de recursos y aseguramientos de insumos de forma priorizada para alcanzar rendimientos estables y elevados.

Las experiencias agrícolas y los rendimientos de este cultivo en el país pueden ser catalogados históricamente como buenos, a excepción de los últimos años en los cuales se aprecia una disminución progresiva de la producción del tubérculo, según datos de FAOSTAT (2016) de 130 933,00 t cosechadas en el 2012 se disminuyó a 53308.00 t en el 2014. Destacando entre las causas los efectos decisivos de la variabilidad climática.

La ubicación tropical e insularidad de Cuba, limitan la extensión temporal de condiciones climáticas idóneas para el cultivo de esta especie, no obstante se aprovechan aquellos períodos correspondientes a la época poco lluviosa con temperaturas menos elevadas (meses de noviembre - abril). Sin dejar de tener en cuenta los riesgos de afectación por la propia variabilidad climática.

Respecto a los efectos que el cambio climático puede generar en este cultivo, Gutiérrez \& Centella (2001) plantean que de no producirse la fertilización por $\mathrm{CO}_{2}$ y de la manifestación de una sensibilidad climática considerada, los rendimientos agrícolas de este cultivo pueden llegar afectarse en un rango de un $40-45 \%$ en Cuba. Otro aspecto significativo a considerar radica en la incidencia de enfermedades capaces de provocar fuertes pérdidas de no contar con una esmerada atención fitosanitaria. Entre ellas, el Tizón tardío causado por Phytophthora infestans (Mont.) de Bary, es una de las enfermedades más desbastadoras de la papa a nivel mundial (Pérez \& Forbes 
2008); capaz de ocasionar daños demoledores que imposibilitan la comercialización y consumo de los tubérculos afectados. Desde el punto de vista agronómico, causa una disminución en la productividad del cultivo así como grandes pérdidas económicas, lo que hace necesario un control temprano del avance de la enfermedad para evitar o reducir estos daños (Lees et al. 2012). Finalmente implica un problema de seguridad alimentaria, entendida ésta como la falta de disponibilidad del alimento para la población que depende de sus necesidades nutricionales.

Esta enfermedad ocupa un lugar principal en Cuba dada lo nociva que resulta para la plantación por los daños que puede acarrear en corto plazo (caída del rendimiento productivo por las severas pudriciones en plantas y tubérculos) (Hernández \& Gómez 2005) por su carácter epidémico (Andréu \& Gómez 2007 citado por Yanes et al. 2012). También Saucedo et al. (2010) catalogan a esta patología como una de las decisivas a la cual se debe prestar esmerada atención fitosanitaria para lograr la producción de papa, como ocurre en zonas de cultivo de la provincia Villa Clara.

Desde las décadas de 1970, 1980 y 1990, trabajan en Cuba, el Instituto de Sanidad Vegetal, la Universidad Martha Abreu de Villa Clara y otras instituciones científicas en la elaboración y aplicación de sistemas de pronóstico para predecir la aparición y desarrollo de Tizón tardío. Prueba de ello radica por citar algunos, los métodos de: "Naumova modificado" de Goméz et al., (1999) y el "Umbral de lluvias" (Padrón 1982), este último sin aplicación hasta la campaña de 19931994 en que comienzan su utilización las Estaciones Territoriales de Protección de
Plantas de Matanzas y La Habana (Gómez et al. 2003a). Ambos sistemas demostraron en su validación buenos índices de confiabilidad que han permitido el manejo efectivo de la enfermedad en determinadas áreas paperas del país (Gómez et al, 2002, Gómez et al. 2003a, Gómez et al. 2003b, Gómez et al. 2003c, Gómez \& Hernández 2003), e integran el Instructivo Técnico que rige la producción de papa en la actualidad.

Basados en la asociación de la incidencia de las variables meteorológicas ajustadas a los requerimientos biológicos del fitopatógeno, en este caso de $P$. infestans, la aplicación de estos métodos predictivos y los éxitos prácticos obtenidos a pesar de sus inexactitudes o insuficiencias, validan su uso y dan evidencias de la importancia que revisten en la interpretación y predicción del comportamiento de esta enfermedad.

Los anteriores criterios y las consideraciones expuestas justifican la necesidad de realizar investigaciones que aporten elementos sobre el comportamiento futuro de los organismos nocivos. Por ello, el objetivo de este trabajo consiste en: Interpretar escenarios bioclimáticos de Phytophthora infestans, agente causal del Tizón tardío de la papa en áreas del cultivo en Ciego de Ávila durante el período de noviembre - abril para los años 2025 y 2030 bajo escenarios (SRES) A2 y B2. A su vez permitirá trazar políticas de gestión dentro de la producción agrícola, teniendo en cuenta que en la actualidad se aboga por una agricultura climáticamente inteligente, capaz de sustentar una producción sostenible del cultivo con un adecuado manejo fitosanitario y el enfrentamiento al cambio climático. 


\section{Materiales y Métodos}

Se confeccionaron escenarios bioclimáticos a partir de fenoclimatogramas que representan valores diarios de: temperatura media (Tmed) y precipitación (Pre) del período en estudio: noviembre - abril (período del cultivo en el país) de los años 2025 y 2030. Para ello se tuvo en cuenta los indicadores de uno de los modelos predictivos vigentes que se instrumenta en Cuba para la predicción de la aparición y el desarrollo de epifitotias de Tizón tardío (P. infestans). En este caso se utilizó el método denominado “Umbral de lluvias', propuesto por Padrón (1982), el mismo constituye un sistema de pronóstico que teniendo en cuenta las condicionantes meteorológicas predice momentos óptimos para la aparición de esta enfermedad; el mismo está recomendado por el Instructivo Técnico para la producción de Papa en Cuba (MINAG 2016).

Método de Umbral de lluvias (Padrón 1982):

Indicadores: Se emplea una línea umbral del acumulado de precipitación para lo cual se elabora un gráfico de ejes $\mathrm{X}$ (días de cultivo), Y (acumulado de precipitación), la línea diagonal se conforma mediante la unión de cuatro puntos separados entre sí por un tiempo trascurrido de cuatro semanas y valores fijos de acumulado de lluvias. El primer punto se establece el primero de diciembre con valor de $0 \mathrm{~mm}$; el segundo con $38 \mathrm{~mm}$ el 31 de diciembre; el tercero con $76 \mathrm{~mm}$ el último día de enero y el cuarto con $114 \mathrm{~mm}$ al cierre de febrero. Una vez trazada la línea diagonal se analiza si los acumulados de precipitación en cada punto referido de la línea sobrepasa o no los umbrales de esta variable establecidos en cada uno. Siempre que el acumulado real hasta la fecha sobre pase el valor umbral y la temperatura media sea inferior a $24^{\circ} \mathrm{C}$, se considerará como un estado de tiempo óptimo para la aparición de epifitotias del fitopatógeno.

Los datos de las variables meteorológicas empleadas pertenecen a salidas del Modelo Climático Regional PRECIS CARIBE, ampliamente utilizado en regiones del Caribe (PRECIS CARIBE, 2010, Taylor et al. 2013) con condiciones de frontera del Modelo Global ECHAM 4. Se consideraron escenarios de emisiones (SRES) A2 y B2 para las coordenadas de $22^{\circ} \mathrm{N}$ y $78.5^{\circ} \mathrm{W}$. Este punto se localiza en una importante área de cultivo en el municipio Baraguá, provincia de Ciego de Ávila, donde están ubicadas la Empresa Cultivos Varios " $L a$ Cuba" y Cooperativas de Producción Agropecuaria decisivas en la producción de este tubérculo. Se comprobó también la calidad de los datos según su estacionalidad, en consideración a los criterios Lecha et al. (1994); y valores extremos, más alejados de la media +/- la desviación estándar. La precipitación por ser espacial y temporalmente variable se ajustó mediante la obtención de un coeficiente de corrección bimestral obtenido durante el período base 19922009; lo que permitió el ajuste de los valores predichos. Los datos de precipitación utilizados pertenecen a la estación pluviométrica " $\mathrm{La} \mathrm{Cuba"}$ ubicada a escasos kilómetros de las coordenadas de salida del modelo.

Para la interpretación y análisis de los resultados se tuvo en cuenta las condicionantes agrotécnicas a que se somete este cultivo en las entidades 
productivas y que se encuentran establecidas por el Instructivo Técnico para la producción de papa en Cuba (MINAG 2016). Las mimas se refieren a: época de cultivo noviembre-abril (período poco lluvioso), suelos ferralítico rojos de categorías agroproductivas I y II, atenciones culturales y riego mediante sistemas aspersión con máquinas de pivote central con normas (150-250 $\mathrm{m}^{3} / \mathrm{ha}$ ) y un régimen no menor de 18 riegos por campaña para este tipo de suelo (con buen drenaje interno y externo). De igual forma se valoró la importancia del régimen de riego en el desarrollo fenológico debido a la influencia de la humedad para la planta y el desarrollo de esta enfermedad. En la interpretación de los escenarios se tuvo en cuenta además la duración de los períodos de cada subfase fenológica representada en los escenarios bioclimáticos, así como las restantes consideraciones agrotécnicas que exige el sistema de agricultura para la producción del tubérculo en el país, igualmente partes del Instructivo.

\section{Resultados y discusión}

\section{Interpretación de escenarios bioclimáticos (2025 y 2030).}

Es necesario profundizar en la fluctuación poblacional de los organismos nocivos para trazar los sistemas de manejo y enfrentar los efectos del cambio climático, al respecto existe poco conocimiento de los efectos de estos cambios sobre la dinámica poblacional de cientos de especies de plagas Altieri \& Nicholls (2008). Ante lo cual es ventajosa la interpretación sobre la base de la descripción de los escenarios bioclimáticos por su contribución informativa, elementos que se aportan a continuación.
La predicción de temperatura media y precipitación en los meses noviembre abril para el año 2025 bajo un escenario línea evolutiva $\mathrm{A} 2$, se muestran en la Figura 1. El análisis visual permite comprobar que los valores de precipitación son bajos de forma general; en los meses de diciembre y abril se presentan las mayores acumulados, sobre pasando la línea de umbral en dos días (cinco y seis) del mes de diciembre con 15 y $11 \mathrm{~mm}$ respectivamente. En los restantes días no existe incidencia de acumulados de precipitación que sobre pasen valores umbrales en la línea diagonal.

Respecto a la temperatura (Tmed), se observó que sus valores oscilan de forma general entre 25 y $30{ }^{\circ} \mathrm{C}$ y solo desciende a los $24{ }^{\circ} \mathrm{C}$ durante algunos días de los meses de diciembre y enero, los cuales pueden favorecer la aparición y desarrollo de la enfermedad, de acuerdo a los criterios térmicos que sustentan este método de predicción.

Sin embargo, la coincidencia de precipitación acumulada diaria por encima de la línea umbral y una Tmed por debajo de $24{ }^{\circ} \mathrm{C}$ según criterios de Padrón (1982), no se presentan durante todo el espacio temporal de desarrollo del cultivo para este año. Los únicos días de diciembre en los cuales el acumulado es superior a la línea umbral de lluvia, la Tmed, muestra valores de 27 y $26{ }^{\circ} \mathrm{C}$, lo cual no conforma condiciones ambientales idóneas para la aparición y desarrollo de la epifitotia.

El escenario bioclimático en condiciones de emisión B2 (Figura 1), muestra una amplia similitud con el anteriormente descrito (escenarios de emisión A2). Aunque, los índices de precipitación acumulada aún presentan valores más 
bajos y en ninguno de los días del período de cultivo se sobre pasa la línea umbral. De otra parte la Tmed mantiene una oscilación general para este espacio temporal entre 25 y $30{ }^{\circ} \mathrm{C}$, y solo en enero presenta escasos días inferiores a 24 ${ }^{\circ} \mathrm{C}$. Se puede resumir que las condiciones meteorológicas que presenta este escenario (SRES-B2) no admiten posibilidades desde el punto de vista climático para la aparición y el desarrollo de la enfermedad de Tizón tardío en las áreas de papa durante el año 2025, acorde a los requisitos referidos por Padrón (1982) para la predicción de epifitias de

Tizón

tardío.
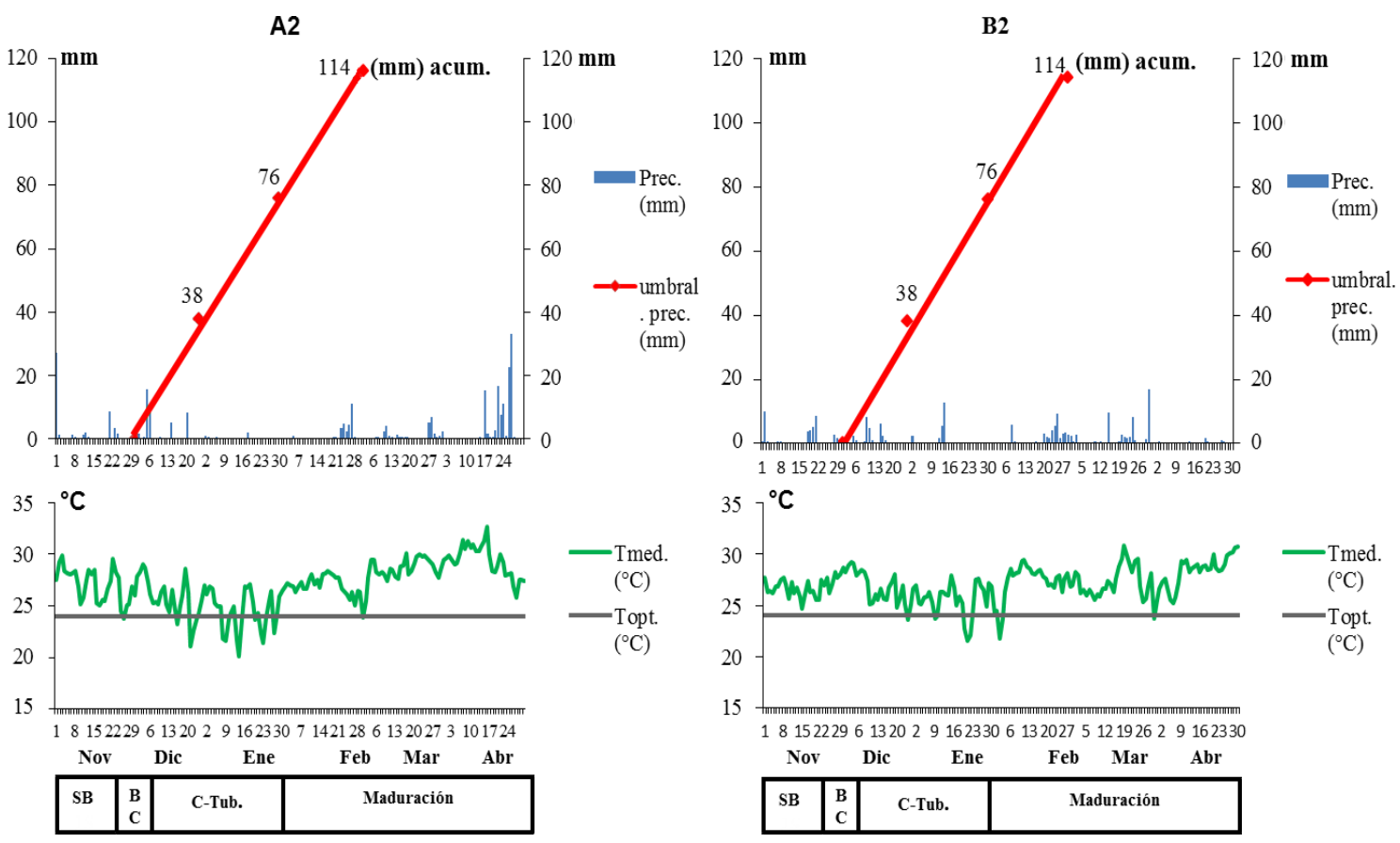

Figura 1. Escenarios bioclimáticos de Tizón tardío para 2025 bajo escenarios de emisiones A2 y B2 valorados por método de Umbral de lluvia. Empresa de Cultivos Varios La Cuba, Ciego de Ávila. Se encuentran representadas la precipitación (barras azules, mm/día), precipitación acumulada (línea roja, $\mathrm{mm} /$ día), temperatura media (línea verde, ${ }^{\circ} \mathrm{C}$ ) y temperatura óptima (línea gris, $24^{\circ} \mathrm{C}$ ).

La Figura 2, muestra los escenarios bioclimáticos A2 y B2 para el año 2030. En el SRES-A2, se predice una precipitación acumulada durante todo el espacio temporal del cultivo con índices Se asume por tanto que la variable precipitación no será favorable para el desarrollo de esta enfermedad. inferiores a la línea de umbral, obsérvese que sus acumulados son bajos y los mayores se registrarán solo en pocos días de forma aisladas durante febrero y abril. 


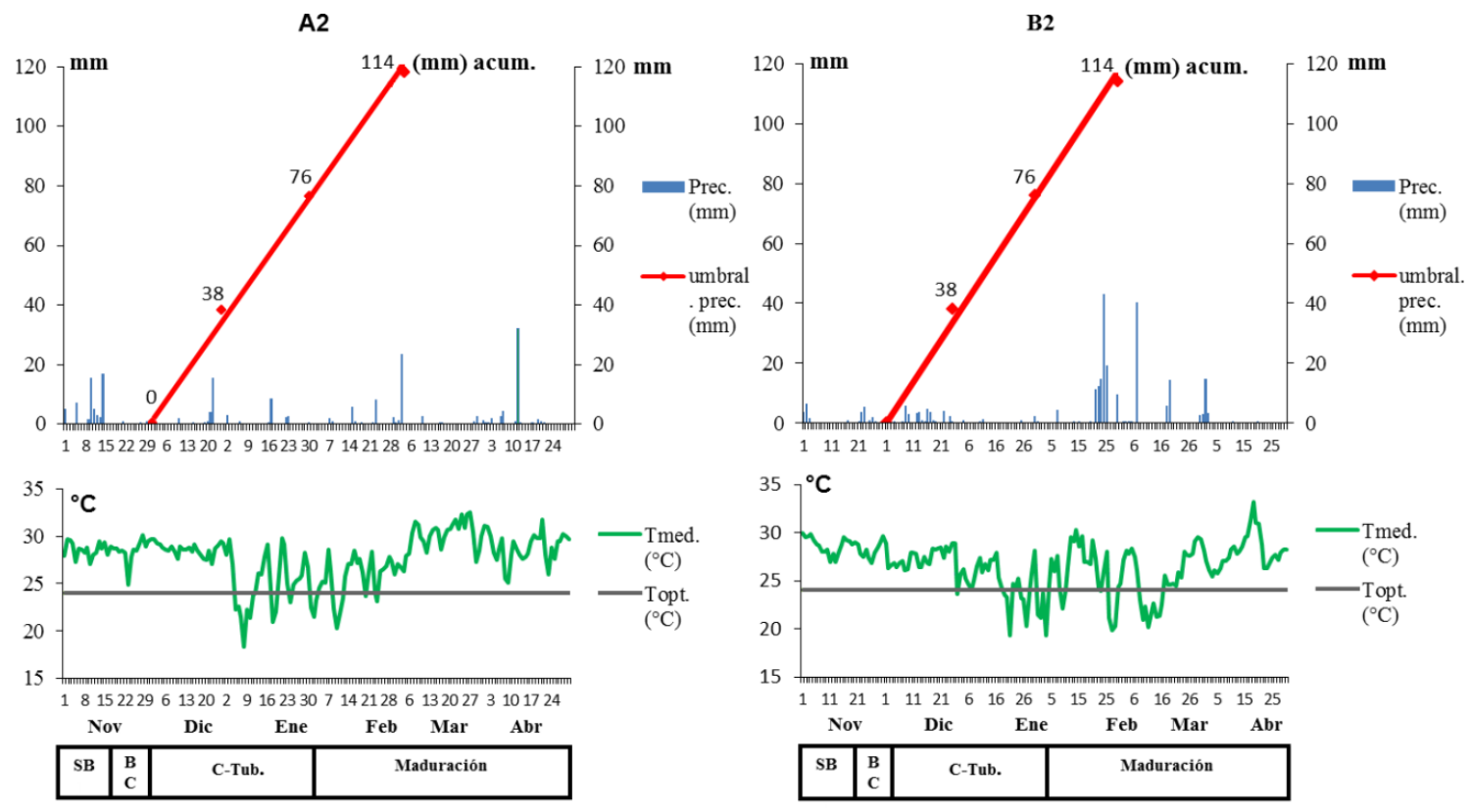

Figura 2. Escenarios bioclimáticos de Tizón tardío para 2030 bajo escenarios de emisiones A2 y B2 valorados por método de Umbral de lluvia. Empresa de Cultivos Varios La Cuba, Ciego de Ávila. Se encuentran representadas la precipitación (barras azules, mm/día), precipitación acumulada (línea roja, mm/día), temperatura media (línea verde, ${ }^{\circ} \mathrm{C}$ ) y temperatura óptima (línea gris, $24^{\circ} \mathrm{C}$ ).

En relación con la Tmed, la variable mantendrá una oscilación entre 25 y 30 ${ }^{\circ} \mathrm{C}$, solo durante los meses de enero y febrero se registran valores por debajo a $24{ }^{\circ} \mathrm{C}$, que no coinciden con los acumulados de precipitación requeridos y que no sobre pasan la línea umbral. Cabe señalar que la combinación de las condiciones meteorológicas previstas por este método de umbral de lluvia no se cumplen, y con ello la ausencia de ocurrencias de procesos epidemiológicos para el Tizón tardío.
En relación al escenario bioclimático bajo el SRES-B2 para el año 2030 (Figura 2), se espera un similar comportamiento de las precipitaciones; los mayores acumulados de esta variable se predicen durante días de febrero y marzo, pero no sobrepasan la línea umbral, lo cual indica que no son volúmenes favorables para el desarrollo del fitopatógeno. La variable Tmed oscila de forma general al igual que en el anterior escenario, salvo algunos 
descensos inferiores a $24{ }^{\circ} \mathrm{C}$ que se pueden apreciar durante el período de enero a marzo, que a diferencia con los anteriores escenarios, muestra una mayor extensión del espacio temporal con temperaturas (más frescas) por debajo de este parámetro. Es coincidente que estos días en que la temperatura es favorable según lo señalado por Padrón (1982), no existirá un acumulado suficiente de precipitación para que se conforme un ambiente favorable para el desarrollo de la epifitia.

La interpretación de los escenarios bioclimáticos descritos anteriormente permiten plantear que existirán condiciones no favorables para el desarrollo de epifitias de Tizón tardío en áreas de Ciego de Ávila, fundamentalmente por los bajos índices de precipitación y los aumentos de temperatura la cual predominará durante el espacio temporal del cultivo con valores medios superiores a $25{ }^{\circ} \mathrm{C}$. Resultados de estudios realizados en Cuba por Vaillant \& Gómez (2009) sobre la presencia del agente causal de Tizón tardío en el cultivo señalan que en los últimos años no se ha informado, debido al incremento de la temperatura y a los amplios períodos de sequía que se han presentado en el país y concluyen que la incidencia de $P$. infestans ha disminuido notablemente en Cuba a causa de los cambios climáticos y el aumento de la temperatura. A pesar de las valoraciones realizadas en atención a los escenarios bioclimáticos en sus diferentes escenarios de emisión A2 y B2 tanto para los años 2025 y 2030 y en consideración a los parámetros del método empleado, es importante reflexionar sobre el efecto que puede ejercer el régimen y la norma de riego de ser suministrado adicionalmente en caso de ocurrencia de precipitación. Esto puede llegar a generar un ambiente más húmedo, favorable y con significación en aquellos lugares del campo donde puedan ocurrir encharcamientos y exceso de acumulación de agua, de existir temperaturas más frescas acordes a los índices del método de Padrón (1982) muy a pesar de considerarse altos por Gómez et al. (2003a), cabe entonces la posibilidad de la aparición de focos de la enfermedad solo de estar presente temperaturas más bajas (temperatura óptima para el desarrollo del fitopatógeno y formación de esporangios que se sitúa entre 18 y $20{ }^{\circ} \mathrm{C}$ durante 8 a 10 h según Smart et al. (2000).

Estos elementos permiten deducir que a pesar de las predicciones se hace inminente cumplir estrictamente las normas establecidas en los instructivos y mantener un monitoreo a las áreas de cultivo que permita el reajuste del manejo fitosanitario in situ.

A pesar de las deducciones realizadas en las interpretaciones de los escenarios bioclimáticos de la no existencia futura de condiciones favorables para el desarrollo de epifitias de Tizón tardío para los años 2025 y 2030 bajo el efecto del cambio climático; se hace preciso referir las conclusiones realizadas por Gómez et al. (2003a) en los que se evalúa la efectividad el método de Umbral de lluvias para esclarecer y aportar argumentos. Estos autores consideran que este método no ofrece exactitud respecto al empleo del parámetro de temperatura media inferior a $24{ }^{\circ} \mathrm{C}$, atribuyen que aún es un índice térmico elevado si se tienen en cuenta los requerimientos térmicos que exige $P$. infestans para desarrollar epidemias, puesto que otros sistemas de predicción empleados en Cuba como el método de Naumova modificado de Gómez et al. (1999) establece otros 
parámetros como: temperaturas mínimas superiores a $11{ }^{\circ} \mathrm{C}$ y máximas superiores a $25{ }^{\circ} \mathrm{C}$ e inferiores a $28{ }^{\circ} \mathrm{C}$, acompañados de humedad relativa mayor de $84 \%$ que permiten predecir, tanto primeras apariciones del fitopatógeno como ocurrencia de epifitotias de una forma más exacta, aunque se reconoce que el umbral de lluvias solo predice la aparición de la epifitia.

Gómez et al. (2003a) al valorar el Umbral de lluvias, argumentan que la no coincidencia más importante de este método se manifiesta cuando alerta la ocurrencia de epidemias y estas no ocurren, debido fundamentalmente a la ausencia de períodos de bajas temperaturas que favorecen el desarrollo del Oomycetes, concluyendo que el umbral de temperaturas medias propuesto no se corresponde con las necesidades térmicas de $P$. infestans para su evolución a epidemia. Agregan además que el fundamento del método está relacionado en el ciclo de vida del patógeno y con la diseminación de la enfermedad, pero que no predice cuándo van a aparecer los primeros síntomas, pues solo pronostica en qué momento la enfermedad se va a presentar epifitóticamente basado en la fuerte relación que existe entre la lluvia y el desarrollo del hongo. A su favor, señalan que el método muestra un porcentaje de coincidencia durante el período que se evaluó de un 71 y 93\%, con un valor medio de $80 \%$.
No obstante e independientemente de este análisis, hay que valorar el desarrollo de $P$. infestans está estrechamente relacionadas con la lluvia, la presencia de agua es necesaria para su conversión en zoosporangios (Blackwell \& Waterhouse 1931, Erwin \& Ribeiro 1996). Elemento fundamental para la dispersión de la enfermedad.

\section{Conflictos de intereses}

Los autores han cumplido las normas éticas de publicación, y no generan conflicto de interés en la presente investigación.

\section{Agradecimientos}

Se agradece al Centro de Meteorología Agrícola y al Centro de Física de la Atmósfera del Instituto de Meteorología el acceso a las salidas del Modelo Climático Regional PRECIS - CARIBE. En especial a los investigadores Dr. Oscar Solano Ojeada y MSc. Ramsés Vázquez por su cooperación. 


\section{Referencias citadas}

Altieri, M. A.; Nicholls, C. I. (2008). Los impactos del cambio climático sobre las comunidades campesinas y de agricultores tradicionales y sus respuestas adaptativas. Agroecología.3: 7-24.

Blackwell, E. M.; Waterhouse, G. M. (1931). Spores and Spore Germination in the Genus Phytophthora», Trans. Br. Mycol. Soc. Inglaterra.15: 294-310.

Cline, W. R. (2007). Global Warming and Agriculture. Impact Estimates by Country, Washington D.C., Center for Global Development/Peterson Institute for International Economics. 96 p.

Erwin, D. C.; Ribeiro, O. K.1996. Phytophthora infestans (Mont.) de Bary (1876). p. 346-353 In: Phytophthora diseases worldwide. American Phytopathological Society, St. Paul, Minn. USA. 562 p.

FAOSTAT. (2016). Organización de las Naciones Unidas para la Alimentación y la Agricultura. Dirección de Estadísticas [Internet]. Roma: FAO c2015-. [Citado 24 Oct 2016]. FAOSTAT Descargar Datos; [aprox 2 p.]. Disponible en: http://faostat3.fao.org/download/Q/QC/S

Ghini, R.; Bettiol; W.; Hamada, E. (2008). Diseases in tropical plantation crops as affected by climate change: current knowledge and perspectives. Plant Pathol. 60:122-123.

Gómez, G.; Rodríguez, J.; Castellanos; L.; González, M. L.; Figueroa, I.; Sarmientos, A, Alujas, D.; Martin, E.L. (1999). Naumova modificado: ajuste de un método de pronóstico para el tizón tardío de la papa y el tomate en Cuba. Rev. FITOSANIDAD. 3(3): 95-100.

Gómez, G.; Suárez, M.; Figueroa, M.; Rivero, T.; Hernández, A. (2002). Pronóstico del Tizón tardío (Phytophthora infestans (Mont.) de Bary de la papa en Cuba. II. Evaluación de la efectividad del modelo de Naumova Modificado. FITOSANIDAD. 6: 35- 38.

Gómez, G.; Suárez, M.; Suárez, I.; Montero, J.; Arredondo, M. E.; Rivero, T.; Hernández, A.; Díaz, E.; Martínez, E. (2003a). Pronóstico del Tizón tardío (Phytophthora infestans (Mont.) de Bary) de la papa en Cuba. V. Evaluación de la efectividad del Método Umbral de lluvias para la predicción de epifitotias. Fitosanidad. 7(3):33-37 [Citado: 12/enero/2016] Disponible en: http://www.redalyc.org/articulo.oa?id=20 $\underline{9118166007}$ 
Gómez, G.; Suárez, M.; Figueroa, M.; Castellanos, L.; Álvarez, C.; Pico, V. M. (2003b). Pronóstico del tizón tardío (Phytophthora infestans (Mont.) de Bary) de la papa en Cuba. III Relación entre los frentes fríos, la ocurrencia de períodos favorables y la aparición de los primeros brotes de la enfermedad. Fitosanidad. 7(1):43-49. [Citado: 01/enero/2016] Disponible en: http://www.redalyc.org/articulo.oa?id=20 $\underline{9118077007}$

Gómez, G.; Suárez, M.; Suárez, I.; Montero, J.; Arredondo, M. E.; Rivero, T.; Hernández, A., Díaz, E.; Martínez, E. (2003c). Pronóstico del Tizón tardío (Phytophthora infestans (Mont.) de Bary) de la papa en Cuba. IV. Estudio de los períodos favorables para el desarrollo de la enfermedad y su relación con el clima. Fitosanidad. 7(2):63-66 [Citado: 12/enero/2016] Disponible en: http://www.redalyc.org/articulo.oa? $\mathrm{id}=20$ $\underline{9118166007}$

Gómez, G.; Hernández, K. (2003). Forecasting Models for Potato Late Blight Management in Cuba. Global Initiative on Late Blight (GILB), Newsletter. International Potato Center. May, (19):13. [Citado fecha: 12 de mayo del 2016]. Disponible en: www.cipotato.org/gilb
Gutiérrez, T.; Centella, A. (2001). Vulnerabilidad y adaptación al cambio climático. En: Centella, A.; Llanes, J.; Paz, L. Primera Comunicación Nacional a la Convención Marco de las Naciones Unidas sobre Cambio Climático. Instituto de Meteorología (INSMET). La Habana. Cuba, 169 p.

Hernández, K.; Gómez， G. (2005). Aplicación de Marcadores bioquímicos y moleculares en poblaciones de Phytophthora infestans (Mont.) de Bary causante del tizón tardío en papa y tomate. Fitosanidad. 9(4): 50.

Intergovernmental Panel Climate Change (IPCC). (2014). Cambio climático: Informe de síntesis. Contribución de los Grupos de trabajo I, II y III al Quinto Informe de Evaluación del Grupo Intergubernamental de Expertos sobre el Cambio Climático [Equipo principal de redacción, R.K. Pachauri y L.A. Meyer (eds.)]. IPCC, Ginebra, Suiza, 157p.

Juroszek, P.; Tiedemann, A. V. (2013). Plant pathogens, insect pests and weeds in a changing global climate: a review of approaches, challenges, research gaps, key studies and concepts. Journal of Agricultural Science.151: 163-188, doi:10.1017/S0021859612000500. 
Lamichhane, J. R.; Barzman, M.; Booij, K.; Boonekamp, P.; Desneux, N.; Huber, L.; Kudsk, P.; et al. (2014). Robust cropping systems to tackle pests under climate change. A review. Agron Sustain Dev. 35: 443-459. doi:10.1007/s13593014-0275-9.

Lecha Estela, L. B.; Paz Castro, L. R.; Lapinel Pedroso, B. P. (1994). El clima de Cuba. Editorial Academia. La Habana.186 p.

Lees, A. K.; Sullivan, L.; Lynott, J. S.; Cullen, W. (2012). Development of a quantitative real-time PCR assay for Phytophthora infestans and its applicability to leaf, tuber and soil samples. Plant Pathology. 61: 867-876.

Luck, J.; Spackmand, M.; Freemand, A.; Tre bickid, P.; Griffithsce, W.; Finlayac, $\mathrm{K}$;

Chakrabortycf, S. (2011). Climate change and diseases of food crops. Plant Pathology. 60: 113-121, doi: 10.1111/j.1365-3059.2010.02414.x.

Ministerio de la Agricultura (MINAG). Dirección de Agricultura. República de Cuba. (2016). Instructivo Técnico para la producción de papa en Cuba. Editorial Ministerio de Agricultura. La Habana. 62p.
Nelson, G. C., Rosegrant, M.W.; Koo, J.; Robertson, R.; Sulser, T.; Zhu, T.; Ringler, C.; Msangi, S.; Palazzo, A.; Batka, M.; Magalhaes, M.; ValmonteSantos, R.; Ewing, M.; Lee, D. (2009). Climate change: Impact on agriculture and costs of adaptation. Food Policy Report 21. International Food Policy Research Institute (IFPRI). Washington, D.C. EEUU. 30p.

Padrón, S. J. (1982). Umbrales de lluvia para el pronóstico del Tizón tardío en Cuba. Revista Ciencia y Técnica en la Agricultura. Serie Protección de Plantas. $5(2): 77-85$.

Pérez, W.; Forbes, G. (2008). El Tizón tardío de la papa. Manual Técnico. Centro Internacional de la Papa (CIP), Lima, Perú. 41p. [citado: abril 2016]. Disponible en: www.cipotato.org/gilb

PRECIS CARIBE. (2010). Proyectando el Cambio Climático en el Caribe con el Modelo Climático HADRMC. Instituto de Meteorología. República de Cuba. En: WEB PRECIS CARIBE 2010.5. [Citado: 12 de enero del 2015]. Disponible en: http://precis.insmet.cu/html/Precis-

Caribe.html.

Rosenzweig, C.; Casassa, G.; Karoly, D. J.; Imeson, A.; Menzel, A.; Rawlins, S.; 
Root, T.L., Seguin, B., Tryjanowski, P. (2007). Assessment of observed changes and responses in natural and managed systems. En Parry ML, Canziani OF, Palutikof J P, van der Linden PJ, Hanson $\mathrm{CE}$, editores. Climate Change: Impacts, Adaptation and Vulnerability. Contribution of Working Group II to the Fourth Assessment Report of the Intergovernmental Panel on Climate Change [Internet]. Cambridge: Cambridge University Press; 2007 p. 79131. [citado 10 Abr 2016]. Disponible en: http://www.ipcc.ch/pdf/assessmentreport/ar4/wg2/ar4-wg2- chapter1.pdf.

Saucedo Castillo, O.; Pérez Vicente, L.; Herrera Isla, L.; Fernández Pérez, L. (2010). Sistema de pronóstico climático del Tizón tardío (Phytophthora infestans) en el cultivo de la papa en la provincia de Villa Clara. Revista electrónica de Veterinaria (REDVET). 11(03B). [Citado: 12/diciembre/2016]. Disponible en:

http://www.veterinaria.org/revistas/redvet $\underline{\text { /n030310B.html }}$

Smart, C. D.; Sandrock, R. W.; Fry, W. (2000). Molecular Techniques and Mystery of the Potato Late Bligth Pathogen. Plant-Microbe Interctions. Edited by Gary Tacey and Noel Keen.
APS (American Phytopathology Society). $336 \mathrm{p}$.

Taylor, M.A.; Centella, A.; Charlery, J.; Bezanilla, A.; Campbell, J.; Borrajero, I.; Stephenson T, Nurmohamed, R. (2013). The précis Caribbean Story: Lessons and Legacies. Bul. Am Meteorol. Soc.; 94(7): 1065-1073.

United Nations Environment Programme (UNEP). (2009). The environmental food crisis - The environment's role in averting future food crises. 30-31p.

Vaillant Flores, D. I.; Gómez Izaquirre, G. (2009). Incidencia de Phytophthora nicotianae y Phytophthora infestans en Cuba. Revista Agricultura Técnica en México. 35(2): 219-223.

Yanes López, N.; Castellanos González, L.; Gómez Brito, R.; Martín Vasallo, C. V.; Morejón López, N. (2012). El pronóstico a corto plazo de Phytophthora infestans (Mont.) De Bary en papa durante 30 campañas en la Empresa Cultivos Varios Horquita. Revista Centro Agrícola 39(2):19-26. 